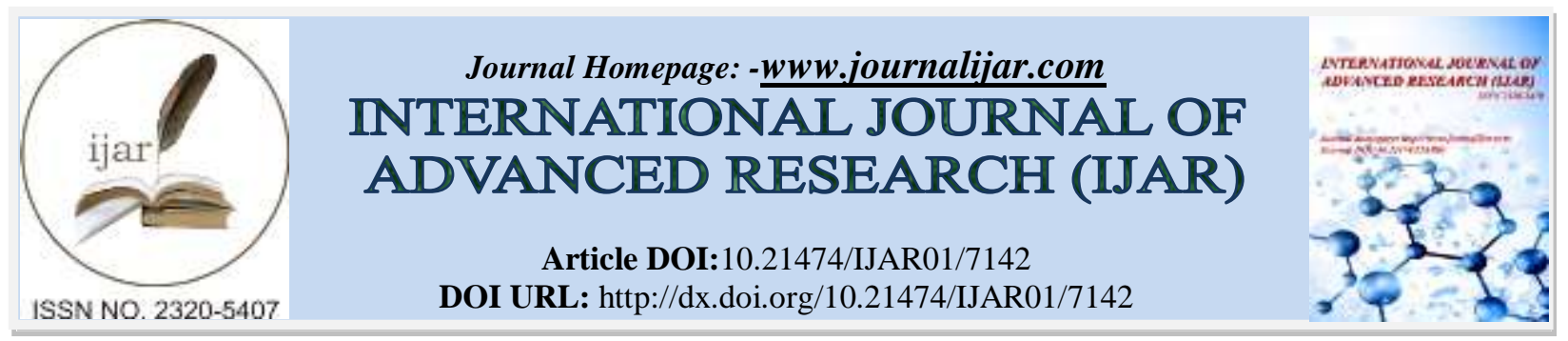

RESEARCH ARTICLE

\title{
A COMPARATIVE STUDY OF MINI-PERCUTANEOUS NEPHROLITHOTRIPSY AND URETEROSCOPIC PNEUMATIC LITHOTRIPSY IN THE TREATMENT OF LARGE IMPACTED UPPER URETERIC STONES.
}

\author{
Mufti Mahmood Ahmed ${ }^{1}$, Bilal Yousuf Mir $^{2}$, Zaffer Saleem Khanday ${ }^{3}$, Syed Javid Farooq Qadri ${ }^{4}$ and Fayaz \\ Ahmad Najar'. \\ 1. Professor Postgraduate Department of Surgery, Government Medical College, Srinagar. \\ 2. Postgraduate Scholar PG Department of Surgery, Government Medical College, Srinagar. \\ 3. Associate Professor Postgraduate Department of Surgery, Government Medical College, Srinagar. \\ 4. Registrar Department of Urology, Government Medical College, Srinagar.
}

\section{Manuscript Info}

Abstract

Manuscript History

Received: 19 March 2018

Final Accepted: 21 April 2018

Published: May 2018

Copy Right, IJAR, 2018,. All rights reserved.

\section{Introduction:-}

The management of large, impacted upper ureteric calculi remains challenging for urologists. Various treatment options include extracorporeal shock wave lithotripsy (ESWL), ureterorenoscopic lithotripsy (URSL), percutaneous nephrolithotripsy (PCNL), laparoscopic and open ureterolithotomy. ESWL has poor overall success rate in the treatment of large stones with a significant possibility of residual fragments. Semi-rigid or flexible ureterorenoscopy with Holmium: YAG laser lithotripsy (URSL) has a stone-free rate of $89-100 \%$ in managing proximal ureteral calculi $^{[1-7]}$. However, large and impacted proximal ureteral stones are difficult to approach. PCNL was introduced as an alternative treatment for large renal and proximal ureteric stones and achieved success in the 1980 s $^{[8]}$. Minimally invasive PCNL (mini-PCNL), which is a modified PCNL using a miniature endoscope by way of a small access tract, can be routinely performed to manage stones in the kidney and proximal ureter ${ }^{[9]}$. PCNL is shown to have a higher success rate compared to other minimally invasive procedures. However, bleeding and fever are the common complications. Conservative management suffices in majority of patients with such complications. Rarely, blood transfusion may be needed. Laparoscopic ureterolithotomy ${ }^{[10,11]}$ is associated with a shorter period of convalescence when compared to an open procedure, but is associated with a higher learning curve. Open ureterolithotomy is indicated for failure of all minimally invasive modalities, in presence of a concomitant open procedure, and the presence of large impacted stone where patients don't consent for multiple procedures ${ }^{[12]}$.

The present study was taken up to analyse two minimally invasive surgical procedures, mini-percutaneous nephrolithotripsy (mini-PCNL) and ureteroscopic pneumatic lithotripsy (URSPL), in the treatment of large $(>15 \mathrm{~mm}$ ), impacted, upper ureteric stones (from PUJ to lower border of L4 vertebra) and to compare the therapeutic outcome, merits and demerits of each procedure.

\section{Material and Methods:-}

After obtaining ethical clearance from the Institutional Ethical Committee, the present prospective, comparative, non-randomised study was conducted in postgraduate Department of General Surgery, Government Medical 
College, Srinagar over a period of two years (December 2014 to December 2016). The study comprised of 60 patients with large $(>15 \mathrm{~mm})$, impacted (diagnosed when there was failure to visualize the ureter distal to a stone with proximal hold up of contrast material as long as 3 hours of excretory urography or stone remaining at the same site in the ureter for more than 2 months or inability to pass guide-wire beyond the stone at initial attempts), upper ureteral (from PUJ to lower border of L4) stones. Group A comprised of 30 patients who underwent mini-PCNL and Group B constituted 30 patients who were subjected to Retrograde Ureteroscopic Pneumatic Lithotripsy. Each patient was allocated to either group A or group B based on his/her own preference with regard to procedure of choice.

\section{Operative technique of mini-percutaneous nephrolithotripsy (mini-PCNL):-}

Under general anaesthesia, patient was placed in lithotomy position. Cystoscopically, ureteric catheterization $(5 \mathrm{~F} / 6 \mathrm{~F})$ was done over a guide-wire. In prone position, a percutaneous puncture was made using a $16 \mathrm{~cm}$ long Cook's $18 \mathrm{G}$ puncture needle by Bull's eye technique. In patients where ureteric catheter could not be negotiated, ultrasonography (USG) - guided puncture was made. The position of the needle was confirmed in the pelvicalyceal system by observing free flow of normal saline through the puncture needle injected from below. The tract was dilated over a 0.035 inch hydrophilic guide-wire using a $16 \mathrm{~F}$ fascial screw dilator. A16F amplatz sheath mounted over a $12 \mathrm{~F}$ nephroscope was introduced through the dilated tract. Stone once identified was fragmented, stone fragments flushed out through the amplatz sheath by infusion of normal saline irrigation. A 5F/6F DJ stent was then placed in. Nephrostomy tube was placed in, when required.

Operative technique of ureteroscopic pneumatic lithotripsy (URSPL):Under spinal anesthesia, with the patient in lithotomy position, ureteroscopy was done using an 8/9.8F semi-rigid ureteroscope. Access to the ureter was made by retrograde insertion of a 0.035 inch floppy tip, hydrophilic guidewire along which the ureteroscope was introduced. The stone was identified and fragmented using a pneumatic Lithoclast. The stone fragments were retrieved. A double-J stent was placed in and removed on an outpatient basis requiring no hospital admission.

\section{Follow-up:-}

Patients with residual fragments in both the groups were followed up for a period of four weeks with X-ray-KUB for assessment of residual stones. The patient was deemed stone free when there was complete clearance of all stone fragments or the presence of fragments $<3 \mathrm{~mm}$, seen on non-contrast CT scan). The procedure was defined as unsuccessful when the procedure was converted into some alternative treatment modality, or the stone could not be reached or fragmented in a single sitting, or fragments $>3 \mathrm{~mm}$ were seen on non-contrast CT scan at 1 month followup.

\section{Statistical Analysis:-}

The recorded data was compiled and entered in a spreadsheet (Microsoft Excel) and then exported to data editor of SPSS Version 20.0 (SPSS Inc., Chicago, Illinois, USA). Continuous variables were summarized in the form of means and standard deviations and categorical variables were summarized as percentages. Student's independent ttest was employed for continuous variables. Chi-square test or Fisher's exact test, whichever appropriate, was used for comparison of categorical variables. Graphically the data was presented by bar diagrams. A P-value of less than 0.05 was considered statistically significant. All P-values were two tailed.

\section{Results:-}

The two studied groups were comparable in terms of demographic parameters viz. age, male to female ratio and stone characteristics [Table 1]. None of the patients had previous history of ESWL. The mean operative time in groups A (mini-PCNL) and B (URSPL) was 89.7+9.63 (range: 66-106) and 59.8+8.89 (range: 42-77) minutes, respectively. The difference was statistically significant ( $\mathrm{p}$ value $<0.001$ ) [Table 2]. The mean hospital stay in groups A (mini-PCNL) and B (URSPL) was 3.4+1.09 and 1.8+0.71 days, respectively. The difference was statistically significant ( $\mathrm{p}$ value $<0.001$ ) [Table 2]. At discharge from the hospital, stone free rates were $86.7 \%$ and $66.7 \%$ in groups A and B respectively, ( $\mathrm{p}$ value $=0.127$ ) [Table 2]. At 1 month follow-up, the stone free rates were $96.7 \%$ in mini-PCNL group and $76.7 \%$ in URSPL group. The difference was statistically significant ( $\mathrm{p}$ value $=0.023$ ) [Table 2]. The success rate was 96.7\% (29/30) in group A (mini-PCNL group) and 76.7\% (23/30) in group B (URSPL group). The difference was statistically significant ( $\mathrm{P}$ value $=0.042$ ) [Table 2]. In our study, a higher number of patients in group A (mini-PCNL) developed post-operative fever and hematuria $(20 \%$ vs. $6.7 \%, \mathrm{p}$ value $=$ 0.254 , and $13.3 \%$ vs. $6.7 \%$, p value $=0.671$, respectively) than in group B [Table 2]. The difference was, however, 
statistically insignificant. One patient in group A required blood transfusion. 5 patients required tube nephrostomy. Two patients in group A in our study developed thoracic complications [Table 2]. One patient developed pneumothorax and another had hydrothorax. Both required intercostal tube thoracostomy. A higher number of patients in group B had stone/fragment(s) migration (13.3\% vs. 3.3\%) [Table 2] and required auxiliary procedures $(10.0 \%$ vs. $3.3 \%)$ [Table 2]. The difference was statistically insignificant. The mean analgesia requirement (in the form of injectable tramadol) in Group A (mini-PCNL group) was found to be significantly more $(168.3+40.45 \mathrm{mg}$ vs. 63.3+29.17, p value <0.001) [Table 2] as compared to Group B (URSPL group), which signifies that the postoperative pain in Group A was significantly more. At 1 month follow-up, patients with residual fragments were reassessed. 1 patient in group A (mini-PCNL group) and 7 patients in group B (URSPL group) had residual stone/fragment(s) more than $3 \mathrm{~mm}$. One patient among them in group B (URSPL group) with difficult fragmentation was posted for another sitting of URSPL and was stone-free post-procedure. Others were followed up to 3 months post-surgery, after which patients were reassessed. Three patients in group B (URSPL group) had clearance without intervention. One patient in group A (mini-PCNL group) with persistant distal ureteral fragment (7 $\mathrm{mm}$ in size) was cleared with URSL and 3 patients in group B (URSPL group) were cleared with ESWL. Therefore, in our study, a higher rate of auxilliary procedures $(10.0 \%$ vs $3.3 \%)$ and retreatment rate $(3.3 \%$ vs. $0.0 \%)$ was noted in group B (URSPL group) as compared to group A (mini-PCNL group) [Table 2]. No loss of follow-up was noted.

Table 1:-Demographic characteristics

\begin{tabular}{|l|l|l|l|}
\hline & Mini-PCNL & URSPL & P value \\
\hline Mean age & $37.5+9.27$ & $37.3+10.54$ & 0.987 \\
& $(22-55)$ & $(21-53)$ & \\
\hline Male to female ratio & $1: 0.67$ & $1: 0.87$ & 0.602 \\
\hline Stone location (R/L ratio) & $1: 1.30$ & $1: 0.87$ & 0.438 \\
\hline Mean stone size & $17.3+2.09$ & $16.8+1.30$ & 0.312 \\
\hline
\end{tabular}

Mini-PCNL $\rightarrow$ mini-percutaneous nephrolithotripsy; URSPL $\rightarrow$ Ureteroscopic pneumatic lithotripsy;

$\mathrm{R} / \mathrm{L}$ ratio $\rightarrow$ right to left ratio

Table 2:-Comparative Results

\begin{tabular}{|l|l|l|l|}
\hline & Mini-PCNL & URSPL & P value \\
\hline Mean operative time & $89.7+9.63$ & $59.8+8.89$ & $<0.001$ \\
\hline Mean hospital stay & $3.4+1.09$ & $1.8+0.71$ & $<0.001$ \\
\hline Success rate & $96.7 \%$ & $76.7 \%$ & 0.023 \\
\hline Stone free rate at discharge & $86.7 \%$ & $66.7 \%$ & 0.127 \\
\hline Stone free rate at 1 month & $96.7 \%$ & $76.7 \%$ & 0.023 \\
\hline Fever & $20.0 \%$ & $6.7 \%$ & 0.254 \\
\hline Prolonged gross hematuria & $13.3 \%$ & $6.7 \%$ & 0.671 \\
\hline Stone migration & $3.3 \%$ & $13.3 \%$ & 0.353 \\
\hline Thoracic complications & $6.7 \%$ & - & 0.492 \\
\hline Ureteral injury & - & $3.3 \%$ & 1.000 \\
\hline Post-op analgesia requirement & $168.3+40.45$ & $63.3+29.17$ & $<0.001$ \\
\hline Auxilairy procedures & $3.3 \%$ & $10.0 \%$ & 0.612 \\
\hline Retreatment & - & $3.3 \%$ & 1.000 \\
\hline
\end{tabular}

Mini-PCNL $\rightarrow$ mini-percutaneous nephrolithotripsy; URSPL $\rightarrow$ Ureteroscopic pneumatic lithotripsy 


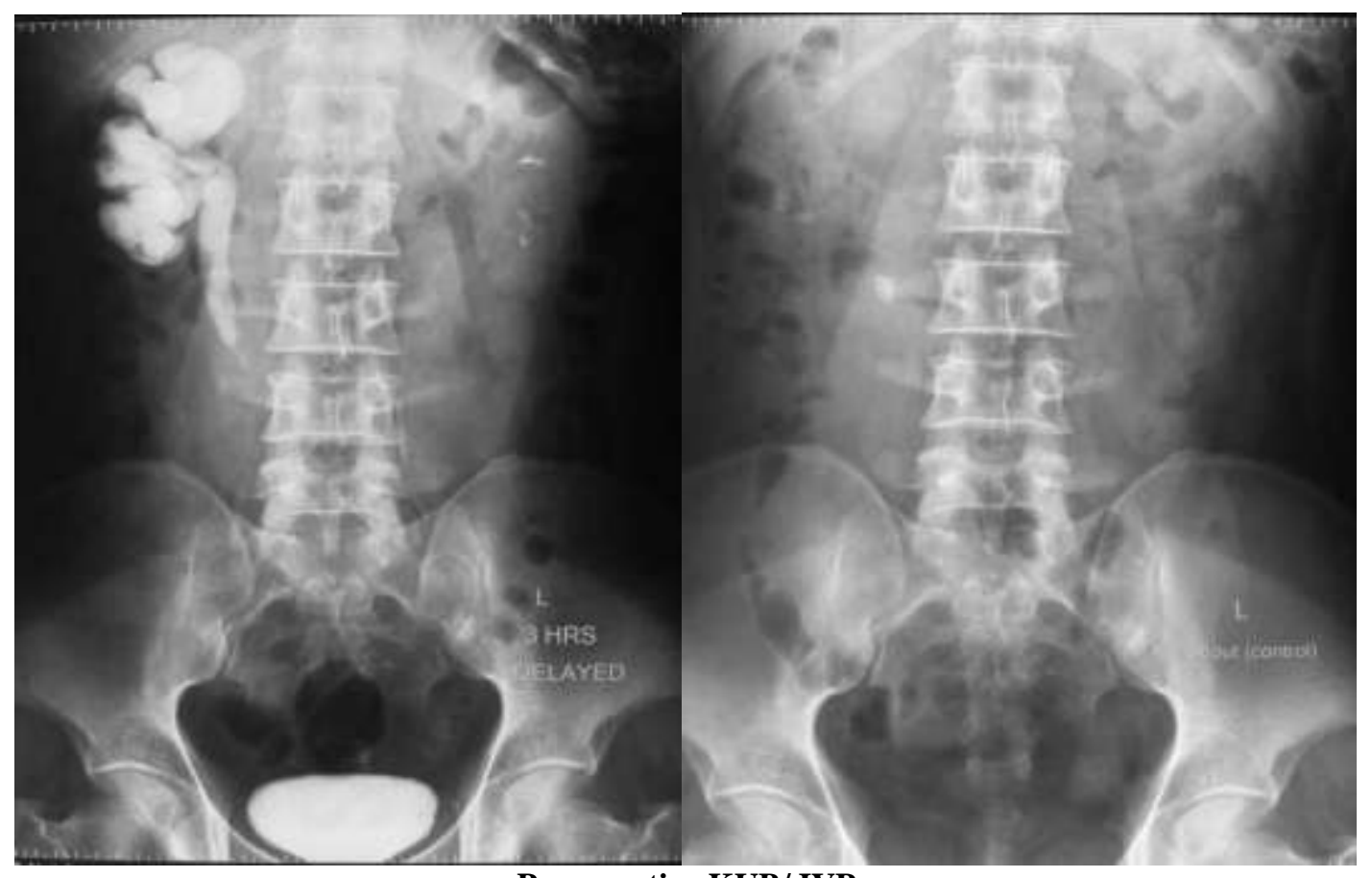

Preoperative KUB/ IVP

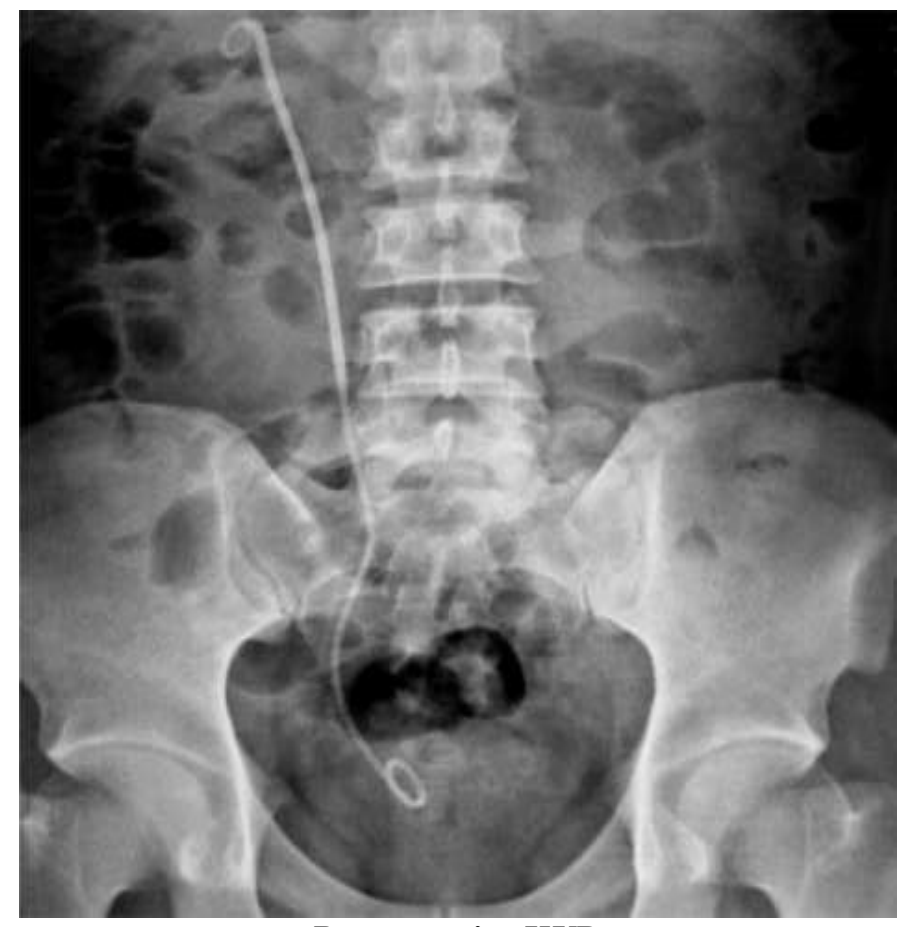

Postoperative KUB

\section{Discussion:-}

Ureteric calculi is a common entity encountered in urology clinics. With increasing size and impactedness, ureteric calculi pose a serious threat to the function of the kidney on the affected side and, thus, the health of the patient. The best treatment option still remains to be determined. Open ureterolithotomy once used to be the standard treatment for impacted, upper ureteric stones. With the advent of lithotriptors, endourology and laparoscopy, less invasive 
procedures are preferred. Both PCNL and retrograde ureteroscopy are accepted treatment modalities for large, impacted, proximal ureteric calculi.

A longer mean operative time, longer mean hospital stay and higher stone clearance rate in antegrade than in retrograde approach for large, impacted, upper ureteric stones has been reported previously ${ }^{[13,14,15]}$. In our study, a significantly longer mean operative time was seen in group A (mini-PCNL group) than in group B (URSPL group) $(89.7 \pm 9.63$ vs $59.8 \pm 8.89$ minutes, $\mathrm{p}$ value $<0.001)$. In our study, the mean hospital stay was significantly longer in group A (mini-PCNL) than in group B (URSPL group) $(3.4 \pm 1.09$ vs $1.8 \pm 0.71$ days, $p$ value $<0.001)$. At discharge from the hospital, stone free rates were $86.7 \%$ and $66.7 \%$ in groups A (mini-PCNL) and B (URSPL) respectively, (p value $=0.127$ ). At 1 month follow-up, in our study, the stone free rates were $96.7 \%$ in mini-PCNL group (group A) and $76.7 \%$ in URSPL group (group B). The difference was statistically significant ( $\mathrm{p}$ value $=0.023$ ). A significantly higher success rate $(96.7 \%$ vs $76.7 \%$, p value $=0.042$ ) was noted in mini-PCNL group (group A) than in URSPL group (group B) in our study. Similar comparisons were observed in various previous studies ${ }^{[14,16]}$. Fever and hematuria are known complications of these two procedures ${ }^{[13,14,17]}$. In our study, a higher number of patients in group A (mini-PCNL group) developed post-operative fever and hematuria $(20 \%$ vs. $6.7 \%$, p value $=0.254$, and $13.3 \%$ vs. $6.7 \%$, p value $=0.671$, respectively) than in group B (URSPL group). The difference was, however, statistically insignificant. One patient in group A (mini-PCNL group) required blood transfusion. Supracostal approach is known to lead to thoracic complications ranging from 5 to $25 \%^{[18,19,20,21]}$. Two patients in group A (mini-PCNL group), in our study, with supracostal approach, developed thoracic complications. One patient developed pneumothorax and another had hydrothorax. Both required intercostal tube thoracostomy. A higher number of patients in group B (URSPL group) had stone/fragment(s) migration (13.3\% vs. 3.3\%) and required auxiliary procedures and retreatment (10.0\% vs. 3.3\%). The difference was statistically insignificant. URSPL has been associated with higher stone retropulsion rate, leading to lower stone-free rate and the need for auxiliary procedures and retreatment ${ }^{[13,14,22]}$. The overall complication rate of mini-PCNL group (43.3\%) was higher than that in URSPL group (30.0\%). The difference was statistically insignificant.

The mean analgesic requirement (in the form of injectable tramadol) in Group A (mini-PCNL group) was found to be significantly more $(168.3 \pm 40.45 \mathrm{mg}$ vs. $63.3 \pm 29.17$, p value $<0.001)$ as compared to Group B (URSPL group), which signifies that the post-operative pain in Group A (mini-PCNL group) was significantly more. More pain and analgesia requirement in antegrade than in retrograde approach for impacted, upper ureteric calculi has been reported $^{[16]}$.

\section{Conclusion:-}

In conclusion, mini-PCNL is a safe and more effective method for the management of large (>15 mm), impacted, upper ureteral stones with a higher success rate and stone free rate. Ureteroscopic Pneumatic Lithotripsy (URSPL) is easier and safer. The operation time and hospital stay are significantly lesser. However, the success rate and stone free rates are lower as compared to mini-PCNL. The need for retreatment and auxiliary procedures is more. 


\section{References:-}

1. Scarpa RM, DeLisa A, Porru D, Usai E. Holmium: YAGlaser ureterolithotripsy. Eur Urol 1999; 35: 233.

2. Dretler SP. Prevention of retrograde stone migration during ureteroscopy. Nat Clin Pract Urol 2006; 3: 60-61.

3. Salem HK. A prospective randomized study comparingshock wave lithotripsy and semirigid ureteroscopy for the management of upper ureteral calculi. Urology 2009; 74: 1216-1221.

4. Preminger GM, Tiselius H, Assimos DG et al. guideline for the management of ureteral calculi. Eur Urol 2007; 52: $1610-1631$.

5. Lam JS, Greene TD, Gupta M. Treatment of proximalureteral calculi: holmium:YAG laser ureterolithotripsy versus extracorporeal shock wave lithotripsy. J Urol 2002; 167: 1972-1976.

6. Preminger GM, Tiselius HG, Assimos DG et al. guideline for the management of ureteral calculi. J Urol 2007; 178: $2418-2434$

7. Araki M, Wong C. Direct comparison of fiberoptic anddigital ureteroscopy for upper urinary tract lithotripsy. J Urol 2007; 4(suppl): V1826.

8. Nahas AR, Eraky I, el-Assmy AM et al. Percutaneous treatment of large upper tract stones after urinary diversion. Urology 2006; 68: 500-504.

9. Li X, He Z, Wu K et al. Chinese minimally invasive percutaneous nephrolithotomy: the Guangzhou experience. J Endourol 2009; 23: 1693-1697.

10. Wickaham JEA. The surgical treatment of renal lithiasis. In: Urinary Calculus Disease. New York: Churchill Livingstone, 1979; pp.145-198.

11. Raboy A, Ferzli GS, Laffreda R, et al. Laparoscopic ureterolithotomy. Urology 1992; 39: 223-25.

12. Ather MH, Paryani J, Memon A, Sulaiman MN. A 10-year experience of managing ureteral calculi: changing trends towards endourological intervention? Is there a role for open surgery? Br J Urol 2001; 88: 173-7.

13. Gu Xiao-jian, Lu Jian Lin, Xu Yan. Treatment of large impacted proximal ureteral stones: randomized comparison of minimally invasive percutaneous antegrade ureterolithotripsy versus retrograde ureterolithotripsy. World Journal of Urology. Dec 2013, Vol. 31, No. 6: 1605-1610.

14. I. H. Bozkurt, T. Yonguc, B. Arslan et al., "Minimally invasive surgical treatment for large impacted upper ureteral stones: ureteroscopic lithotripsy or percutaneous nephrolithotomy?" Canadian Urological Association Journal 2015; Vol. 9, No. 3-4.

15. Stavros Sfoungaristos, Ioannis Mykoniatis, Ayman Isid, Ofer N. Gofrit, Shilo Rosenberg, Guy Hidas et al. Retrograde versus Antegrade Approach for the Management of Large Proximal Ureteral Stones. Bio Med Research International. Jan 2016; Vol. 2016: 1-4.

16. Moufid K, Abbaka N, Touiti D, et. al. Large impacted upper ureteral calculi: A comparative study between retrograde ureterolithotripsy and percutaneous antegrade ureterolithotripsy in the modified lateral position. Urol Ann. 2013;5:140-6.

17. Sun X, Xia S, Lu J, et al. Treatment of large impacted proksimal ureteral stones: Randomized comparison of percutenous antegrade ureterolithotripsy versus retrograde ureterolithotripsy. J Endourol. 2008; 22: 913-7.

18. Lojanapiwat B., Prasopsuk S. Upper pole access for percutaneous nephrolithotomy. Comparison of supracostal and infracostal approaches. J Endourol. 2006; 20: 391-395.

19. Rajiv Yadav, Monish Aron, Narmada P Gupta, Ashok K Hemal, Amlesh Seth, Surendra B Kolla. Safety of supracostal punctures for percutaneous renal surgery International journal of urology. Oct 2006, Volume 13, Issue 10 Pages 1267-1270

20. S.H. Mousavi-Bahar, S. Mehrabi, M.K. Moslemi. The safety and efficacy of PCNL with supracostal approach in the treatment of renal stones. Int Urol Nephrol. 2011; 43: pp. 983-98.

21. Tarek El-Karamany. A supracostal approach for percutaneous nephrolithotomy of staghorn calculi: A prospective study and review of previous reports. Arab Journal of Urology 2012 Dec; Vol. 10, Issue 4: pages 358-366.

22. Hai Li, Wanli NA, Hongyan Li, Yang Jiang, Xinquan Gu, Muchun Zhang et al. Percutaneous Nephrolithotomy Versus Ureteroscopic Lithotomy for Large $(>15 \mathrm{~mm})$ Impacted Upper Ureteral Stones in Different Locations: Is the Upper Border of the Fourth Lumbar Vertebra a Good Indication for Choice of Management Method? Journal of Endourology. Sep 2013; 1120-1125. 